\title{
High-Gain Array Antenna Based on Composite Right/Left-Handed Transmission Line
}

\author{
Ningli Zhu, Quanyuan Feng, Zhi-Ang Wu \\ Institute of Microelectronics, Southwest Jiaotong University, Chengdu, China \\ E-mail: bk20082582@my.swjtu.edu.cn
}

Received August 20, 2011; revised September 19, 2011; accepted September 27, 2011

\begin{abstract}
In this paper a metamaterial-inspired antenna with high gain and good directivity is designed. Based on the concept of composite right/left-handed transmission line (CRLH-TL), the proposed antenna is realized based on three leakage wave unit cell and a left handed circular ring slot incorporated on the surface. The maximum achievable gain at the resonant frequency of $5.6 \mathrm{GHz}$ is $6.933 \mathrm{dBi}$, and the return loss at $5.6 \mathrm{GHz}$ can be $-20 \mathrm{db}$. This proposed design has a simple structure and a compact dimension of $35 \mathrm{~mm} * 40 \mathrm{~mm} * 1 \mathrm{~mm}$, which is suitable for particular wireless communication application such as WiFi and WLAN.
\end{abstract}

Keywords: Composite Right/Left-Handed CRLH, Array Structure, High-Gain

\section{Introduction}

Electromagnetic metamaterials are effectively homogeneous artificial structures engineered to provide electromagnetic properties not readily observable in nature, such as, an index of refraction that may be negative, less than one, or modulated in a graded manner [1]. Particularly, LH metamaterials has made it possible to realize novel microwave applications such as small resonant antennas, dominant-mode leaky-wave antennas, negative refractive index lenses, and dual-band components which are not possible before [2]. The significance of LH metamaterials to both the engineering and scientific communities has sparked lots of formation of international conferences dedicated to metamaterial research, their applications, publication of many books, and so on and so forth.

As the everlasting demand for faster and more compact electronic devices, composite right/left-handed (CRLH) transmission line metamaterials [3], with their rich dispersion information and excellent directivity, have received significant concern and led to numerous novel multifunctional and compact microwave applications over the past decade, especially on antennas. Various practical leaky-wave [4,5] and resonant-type CRLH antennas have been designed, and often the resonant-type antennas offer more alternative properties like multiband operation, zeroth-over high efficiency, good directivity, but suffers from narrow bandwidth. There are a lot of difficulties in achieving a broad bandwidth while keeping a solid ground.

This paper explores the possibility of designing a high gain and directive antenna with a solid ground inspired by the composite right/left-handed (CRLH) transmission line metamaterials concept. All the antenna parameters, such as the return loss, gain, are provided in detail.

\section{Configuration and Working Principle}

\subsection{Configuration}

Figure 1 shows the configuration of the proposed antenna. The antenna is implemented on an $\mathrm{f} 4 \mathrm{~b}-2$ substrate with a thickness of $1 \mathrm{~mm}$ and a relative permittivity of 2.65. It consists of three unit cells as show in the Figure 2. A circle ring notch is made on the metal surface. This antenna is fed by a piece of $50 \mathrm{Ohm}$ microstrip line in the way of edge coupling.

\subsection{Working Principle}

The array structure is essentially a CRLH structure as discussed in [3]. The slot between the unit cells and the circular feed line acts as the left-handed (LH) series capacitor. This via in the unit cell center plays the role of the shunt inductance. They correspond to the LH contribution. The right-handed ( $\mathrm{RH})$ contribution comes from the distributed series inductance and distributed shunt capacitance. 


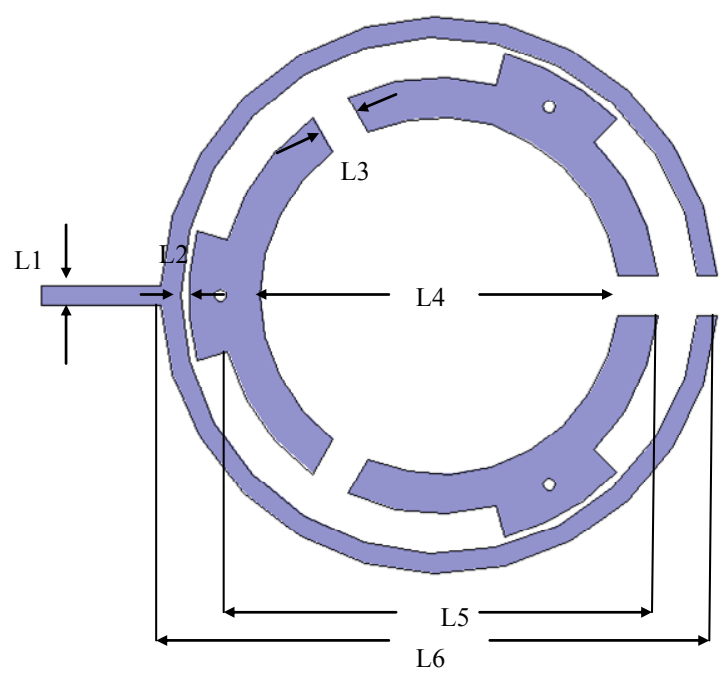

(a)

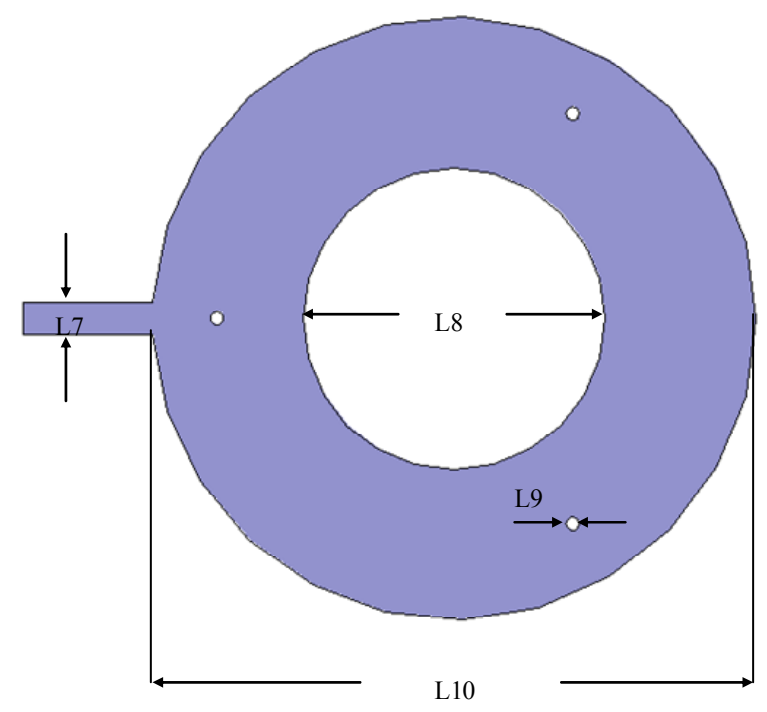

(b)

Figure 1. Configuration of the proposed metamaterial-inspired array antenna [6]. (a) Top view; (b) Bottom view. [7]

The unit impedance and shunt admittance are given by

$$
\begin{gathered}
Z_{s e}=j \omega L_{R}+\frac{1}{j \omega C_{L}}=j \omega L_{R}\left\{1-\left(\frac{\omega_{s e}}{\omega}\right)^{2}\right\} \\
Y_{s h}=j \omega C_{R}+\frac{1}{j \omega L_{L}}=j \omega C_{R}\left\{1-\left(\frac{\omega_{s h}}{\omega}\right)^{2}\right\} \\
\omega_{s e}=1 / \sqrt{L_{R} C_{L}} \\
\omega_{s h}=1 / \sqrt{L_{L} C_{R}}
\end{gathered}
$$

$\omega_{s e}$ is the unit resonant frequency and $\omega_{s h}$ is the parallel resonant frequency. The unit resonance in series

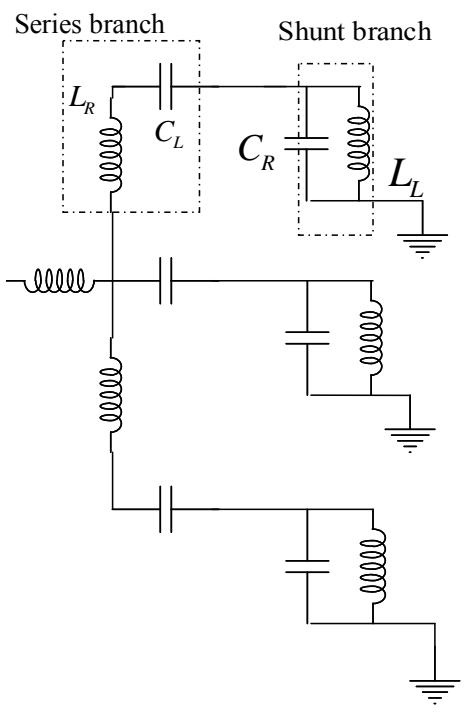

Figure 2. Reactively terminated resonator based on CRLHTL.

branch is the dominant factor when we design the shortended zeroth-order resonators, whereas the parallel resonance in the shunt branch is decisive crux for open-ended zeroth-order resonators [6]. Once these resonant frequencies $\omega_{\text {se }}$ and $\omega_{\text {sh }}$ are the same, there is no bandgap between left-and right-handed passbands in the CRLH-TL, what's more, the CRLH-TL is referred to as a balanced CRLH-TL.

There exhibits a phase delay due to a transmission along the transmission line from Terminall to Terminal 2 and the vice versa way, we define the quantities $\Delta \phi_{+}$ and $\Delta \phi_{-}$to denote this kind of delay, shown in Figure 2. And the reflection at Terminals 1 and 2 will causes a phase shifts, which is represented as $\Delta \phi_{1}$ and $\Delta \phi_{2}$, respectively. In this case, as long as the following phase relation holds [7]:

$$
\Delta \phi=\Delta \phi_{+}+\Delta \phi_{-}+\Delta \phi_{1}+\Delta \phi_{2}=2 n \pi
$$

Then the resonant condition then is satisfied. And also

$$
\Delta \phi_{+}=\Delta \phi_{-}=\beta l
$$

$\beta$ represents the phase constant of the transmission line, $l$ represents the length of the resonator, and $n$ is an integer. If both terminals are open or shorted, relation reduces to [7]

$$
\beta=\frac{n \pi}{l}
$$

The array unit structure exhibits an equivalent circuit as shown in Figure 3(a). The dispersion diagram for this kind of CRLH unit cell is shown in Figure 3(b). The CRLH transmission line supports a fundamental LH wave (phase advance) at lower frequencies and a $\mathrm{RH}$ wave (phase delay) at higher frequencies. 


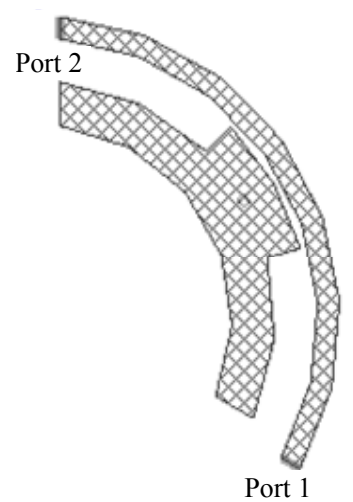

(a)

Series branch

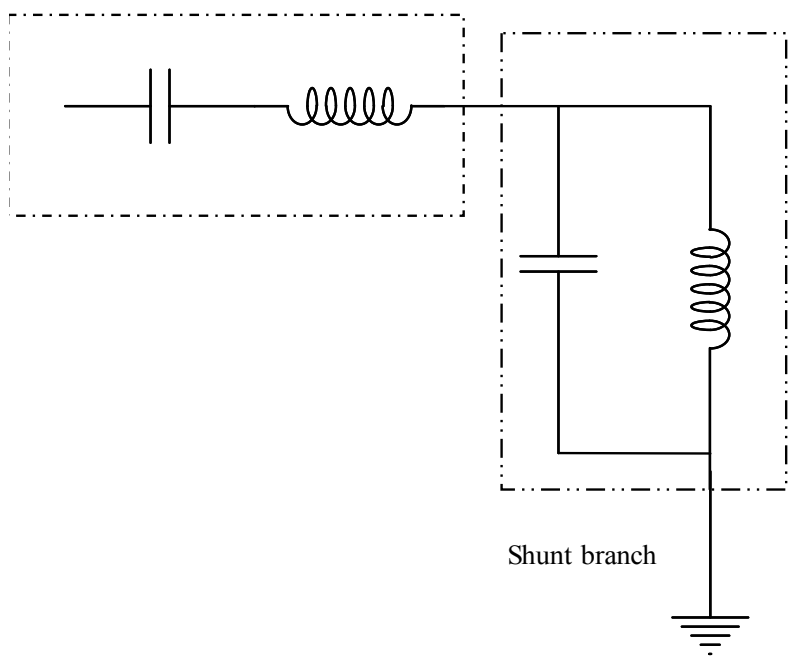

(b)

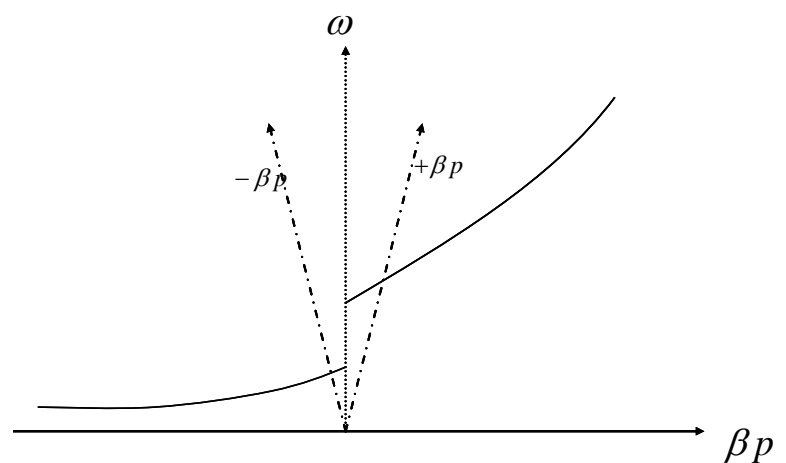

(c)

Figure 3. (a) The array unit cell; (b) Equivalent circuit model for the array unit cell; (c) Its corresponding dispersion diagram.

The ring notch on the surface changes its operating principle. The patch inside the ring slot becomes a new CRLH resonator which gives increased resonance frequencies, especially, for the zeroth order resonance [8].

\section{Simulation Results}

\subsection{Model and Simulate the Antenna}

To analysis the proposed antenna, full wave electromagnetics (EM) software was used to model and simulate the antenna. Figure 4 illustrates the full wave EM model of the proposed antenna. Substrate material with permittivity of 2.65 , thickness of $1 \mathrm{~mm}$ and dielectric loss tangent of 0.001 is used. The geometry parameters of the proposed antenna are shown in Table 1. The simulated result of the return loss of the proposed antenna is shown in Figure 5. As can be seen from Figure 5, the return loss at resonant frequency $5.6 \mathrm{GHz}$ is $-20 \mathrm{db}$. The impedance bandwidth at $-10 \mathrm{db}$ return loss is from $5.5 \mathrm{GHz}$ to $5.565 \mathrm{GHz}$.

\subsection{Simulation}

Figure 6 shows the surface current of this proposed antenna. This Surface Current strength is symmetric and mainly concentrated in the gap and the inductor. The electromagnetic field is coupling and superposing outfield and then changing the directivity of the proposed antenna, thus making the gain achieve a higher value.

Figure 7 shows the simulated result of gain of the proposed antenna. The maximum achievable gain is 6.933 $\mathrm{dBi}$ at the resonant frequency of $5.6 \mathrm{GHz}$. Compared to conventional microstrip antennas, the proposed one possesses a better gain performance due to its CRLH structure. Simulated radiation patterns in each plane are shown in Figures 8-10.

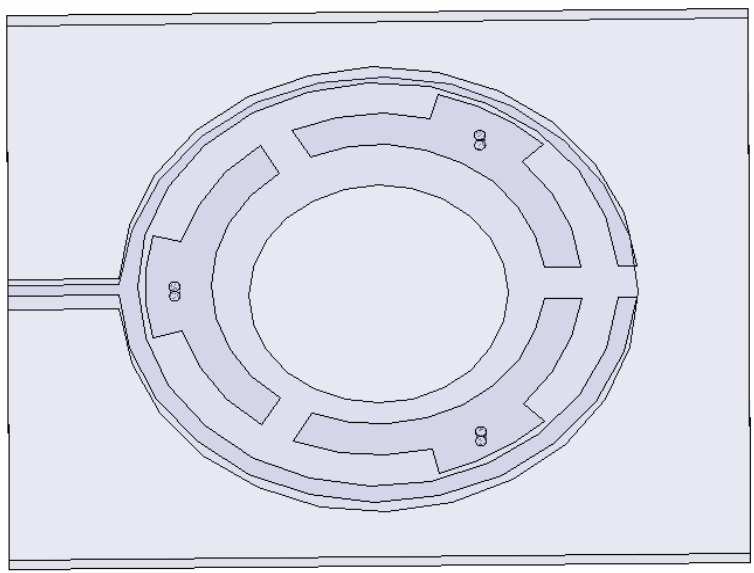

Figure 4. Full wave EM model of the proposed antenna.

Table 1. Critical dimensions of the antenna (Unit: $\mathbf{m m}$ ).

\begin{tabular}{llllllllll}
\hline$L 1$ & $L 2$ & $L 3$ & $L 4$ & $L 5$ & $L 6$ & $L 7$ & $L 8$ & $L 9$ & $L 10$ \\
\hline 1 & 0.4 & 2 & 9 & 11 & 28 & 1.5 & 14 & 0.6 & 28 \\
\hline
\end{tabular}




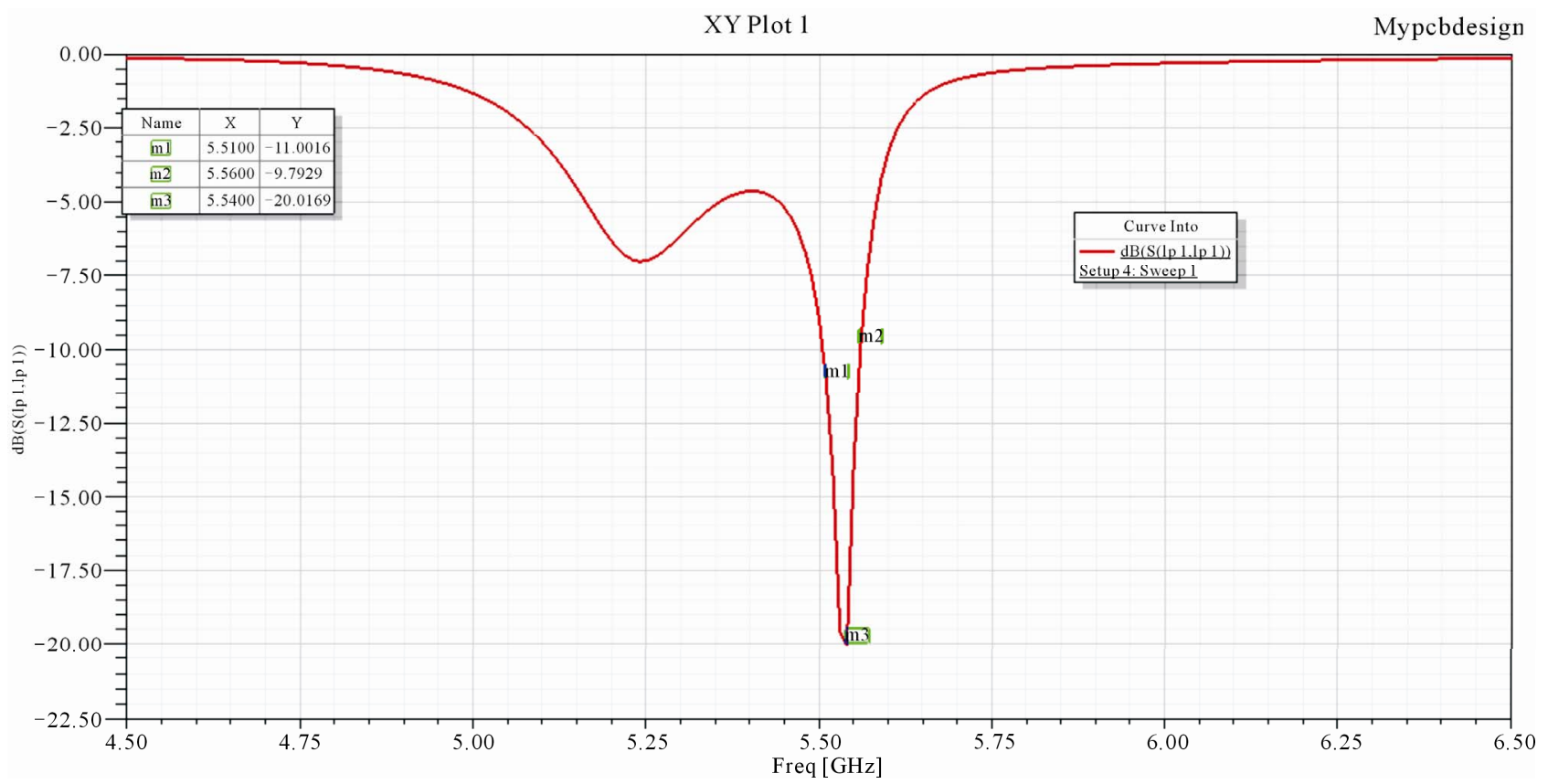

Figure 5. Simulated return loss of the proposed antenna.
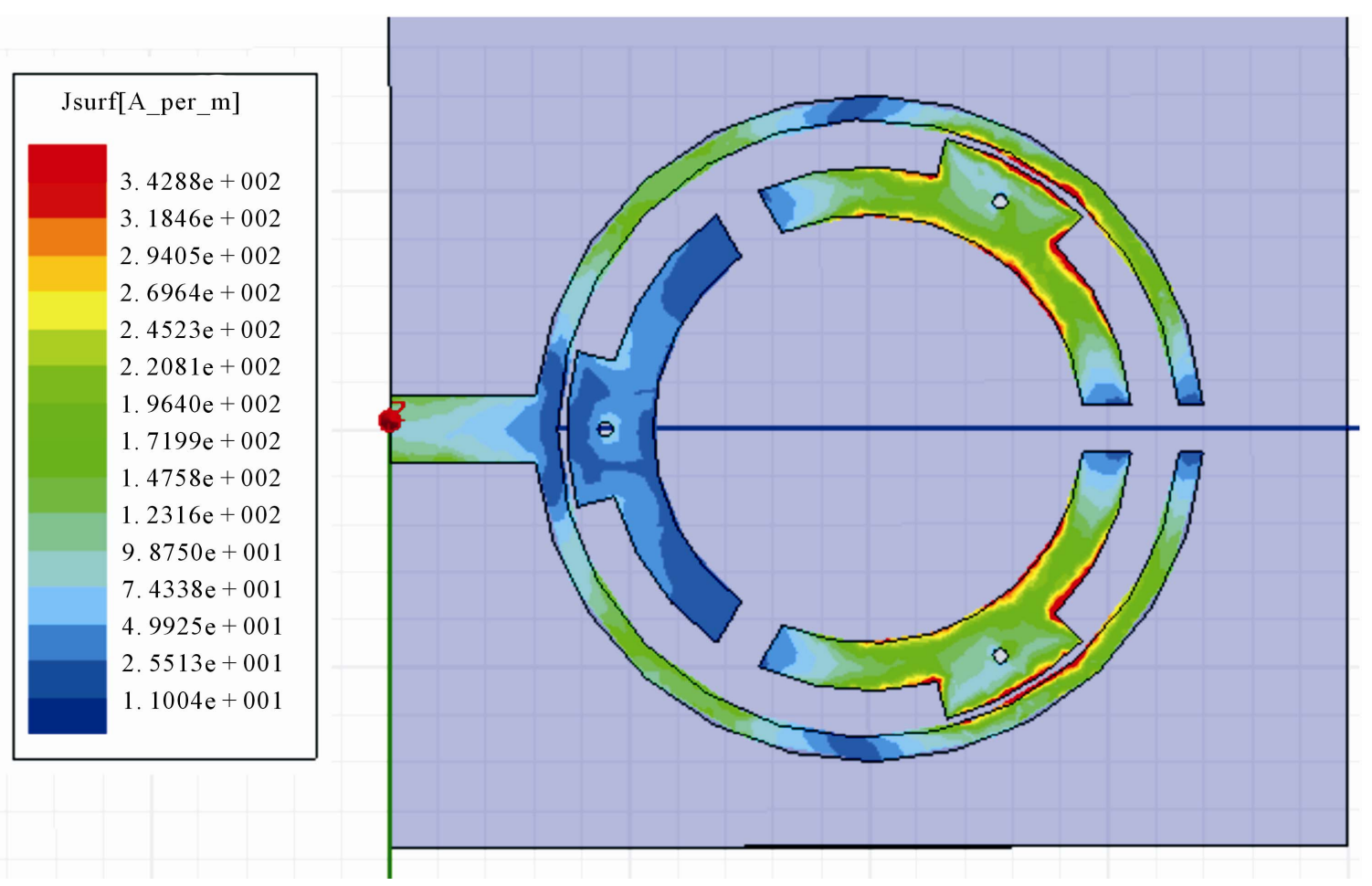

Figure 6. Surface current.

\section{Experiment Verification}

To verify the simulated results, the proposed antenna was fabricated and measured using the $\mathrm{f} 4 \mathrm{~b}-2$ substrate with a thickness of $1 \mathrm{~mm}$. Figure 10 shows the photograph of the fabricated antenna. The measured versus the simu- lated return loss from HFSS for this antenna is shown in Figure 11. The design parameters are also presented below the figure.

Figure 12 shows simulated and measured return loss of the proposed antenna. We can see from the picture, the measured results correspond to the simulation ones. 

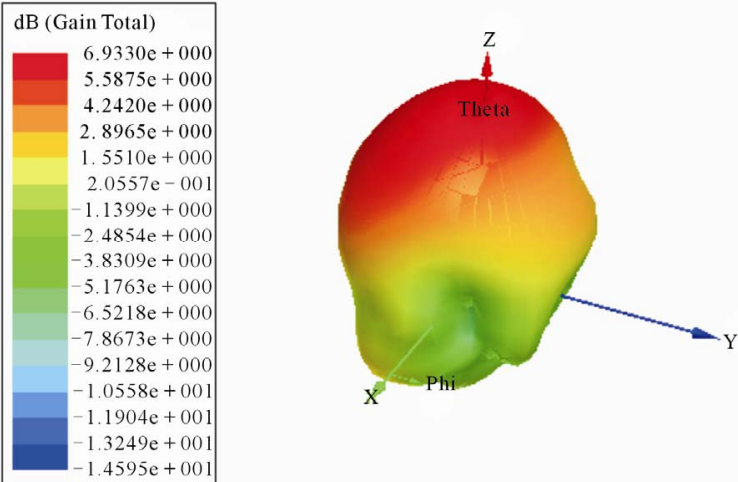

Figure 7. Simulated gain of the proposed antenna.

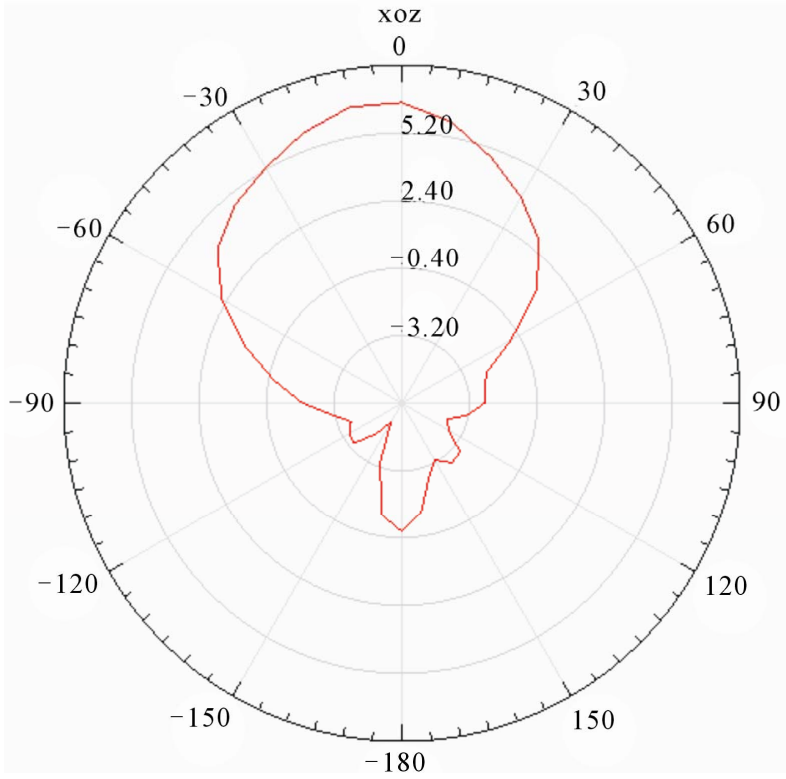

Figure 8. Simulated radiation patterns in the $x-z$ plane.

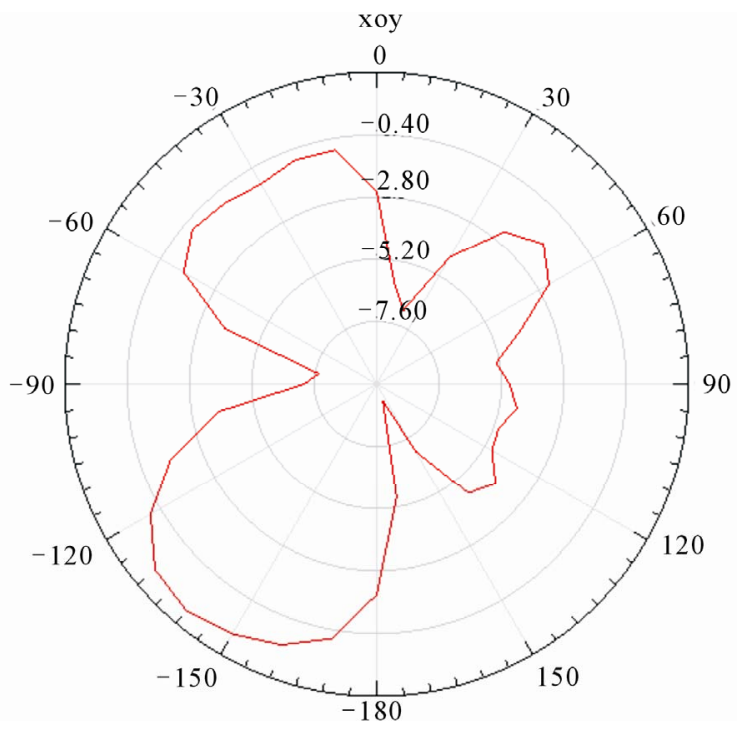

Figure 9. Simulated radiation patterns in the $x-y$ plane.

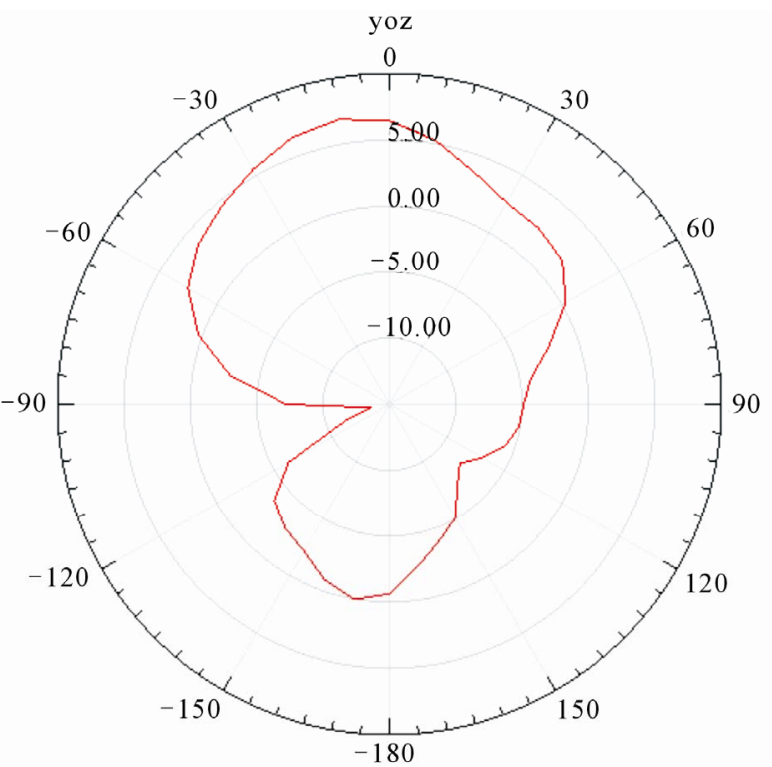

Figure 10. Simulated radiation patterns in the $y-z$ plane.

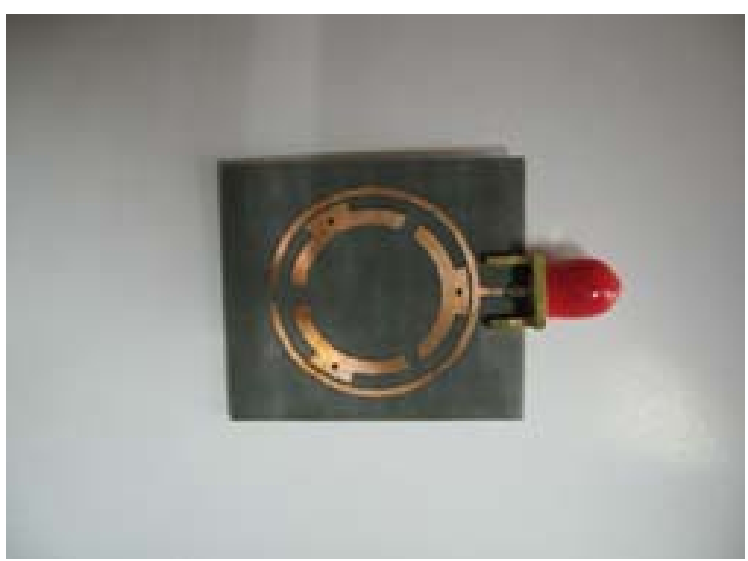

(a)

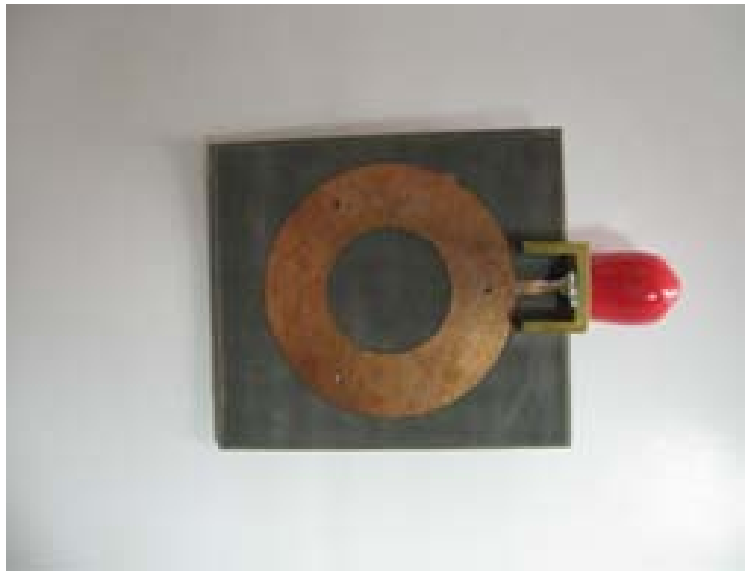

(b)

Figure 11. Photograph of the fabricated antenna inspired by composite right/left handed transmission line. (a) Top view; (b) Bottom view. 


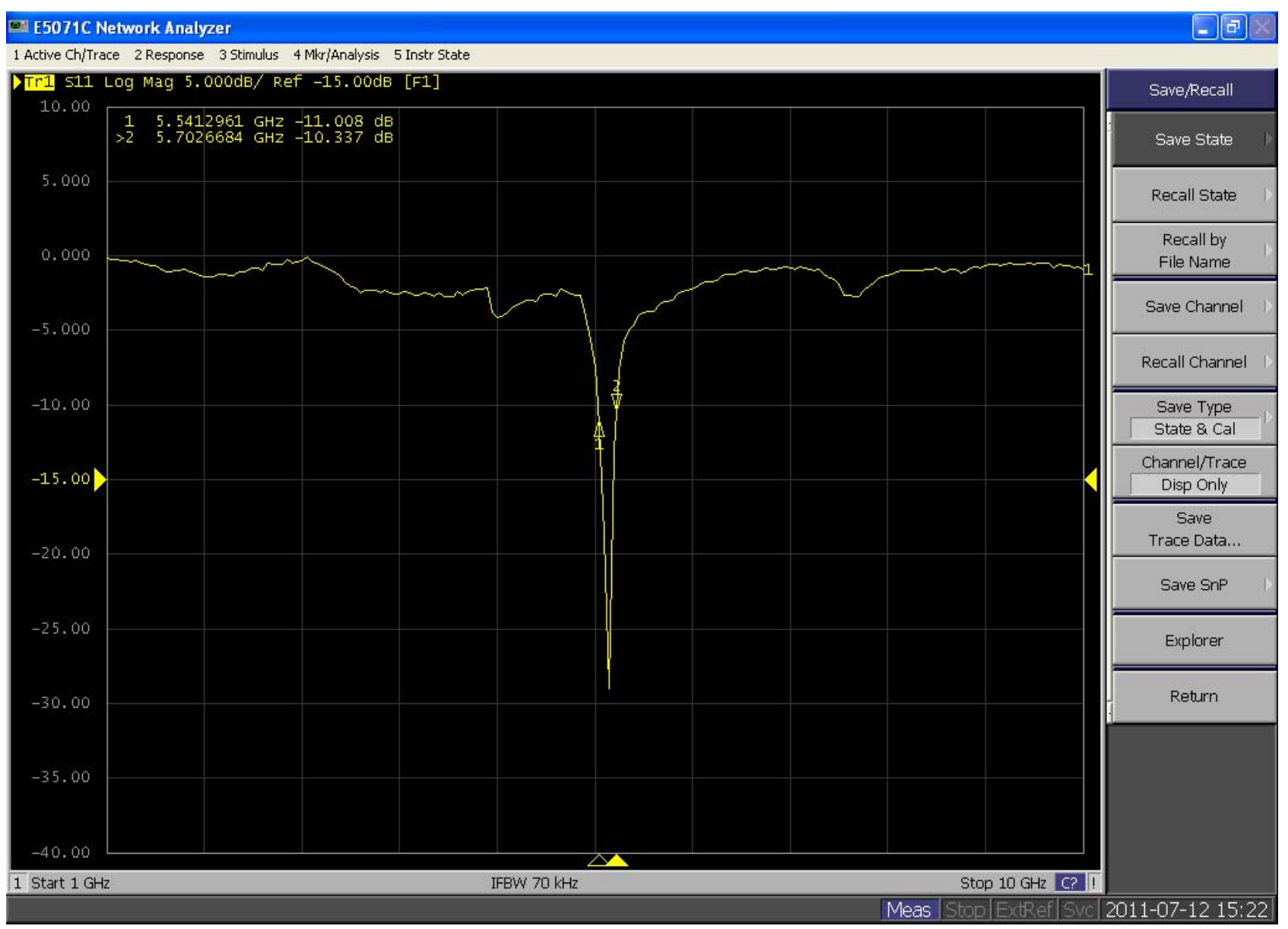

(a)

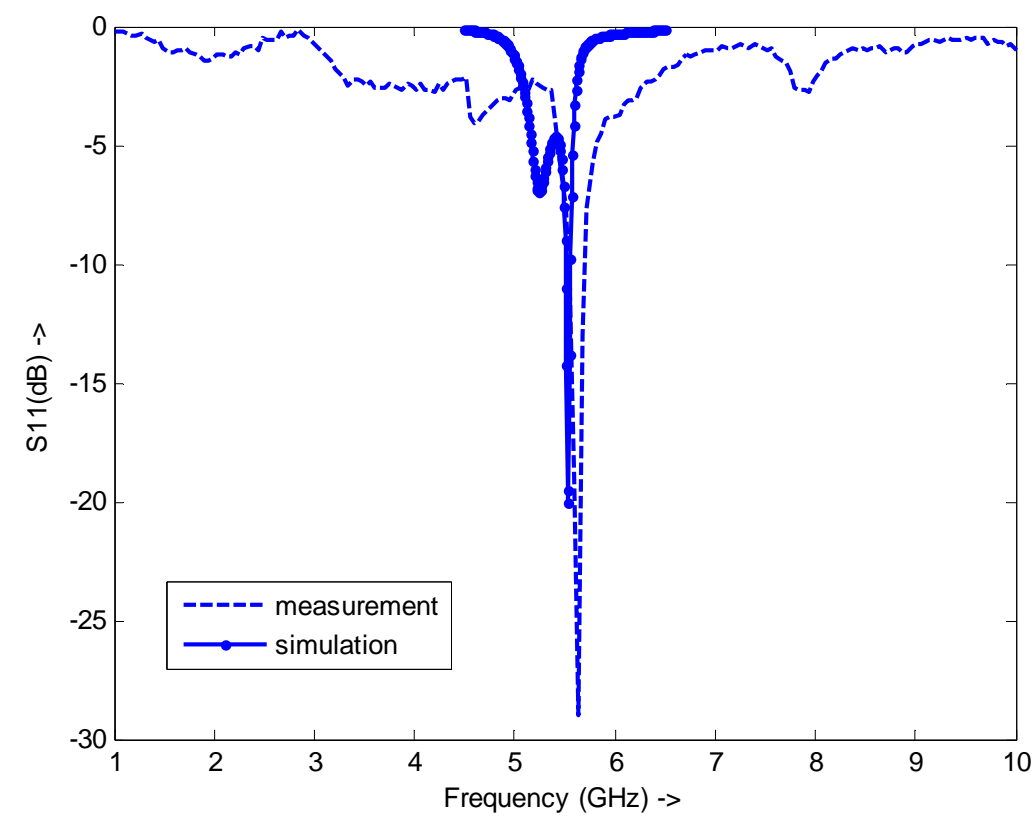

(b)

Figure 12. (a) Measured return loss of the proposed antenna by the Vector network; (b) Simulated and measured return loss. 


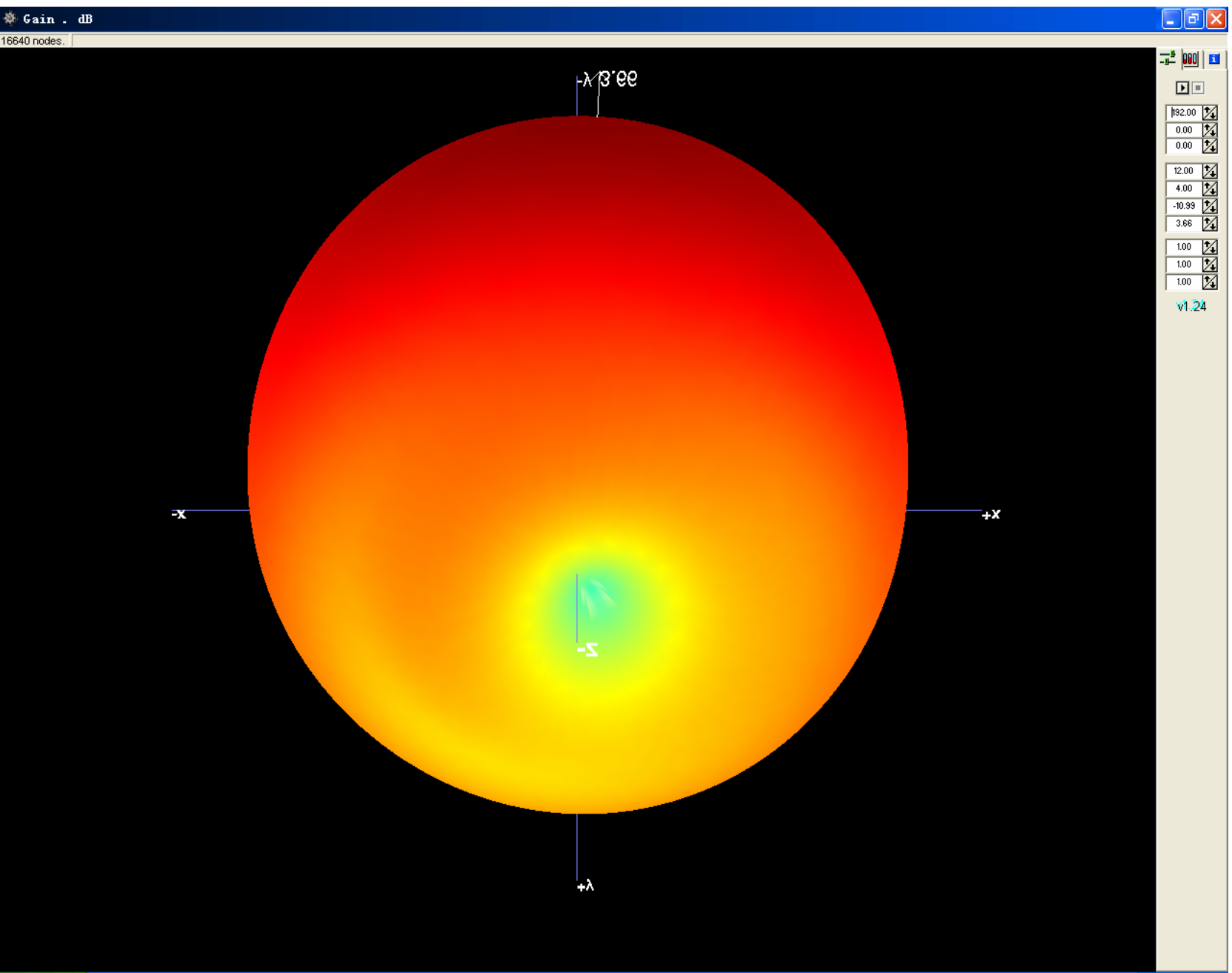

Figure 13. The measured antenna gain is $3.99 \mathrm{dBi}$ at $5.6 \mathrm{GHz}$.

Figure 13 shows the gain for the antenna. It is noted that since the zeroth order resonance is generated by the inner patch inside the slot, the radiation at this frequency mainly goes to the broadside which is different from traditional zeroth order resonance antenna. This is due to the fact that the electrical field exists inside the ring slot which leads to a horizontal polarization. The measured antenna gain is $3.99 \mathrm{dBi}$ at $5.6 \mathrm{GHz}$, a little different with the $6.93 \mathrm{dBi}$ in the simulation. This may due to the limitation of fabrication technology.

\section{Conclusions}

In this paper, a high gain and directive antenna inspired by composite right/left handed transmission line is successfully achieved. We can apply this small array structure for particular wireless communication use such as WiFi and WLAN. A simple circle ring notch is made on the surface which creates an inner CRLH patch and an outside microstrip patch. The working principle of this antenna is provided. Simple structure, high gain and good directivity make this antenna attractive for practical applications.

\section{Acknowledgements}

The work is supported by the National Natural Science Foundation of China (NSFC) under Grant 60990320, 60990323 , and the engineering practice project of Southwest Jiaotong University. The authors would like to thank Qianyin Xiang for his continuous assistance.

\section{References}

[1] C. Caloz, T. Itoh and A. Rennings, "CRLH Metamaterial Leaky-Wave and Resonant Antennas," IEEE Antennas and Propagation Magazine, Vol. 50, No. 5, 2008, pp. 25 39. doi:10.1109/MAP.2008.4674709

[2] C. Kittle, "Introduction to Solid State Physics," 7th Edition, Wiley Text Books, New York, 1964. 
[3] C. Caloz and T. Itoh, "Electromagnetic Metamaterials: Transmission Line Theory and Microwave Applications," Wiley-IEEE Press, New York, 2005. doi:10.1002/0471754323

[4] Y. Dong and T.Itoh, "Composite Right/Left- Handed Substrate Integrated Waveguide and Hald Mode Substrate Integrated," IEEE Transmission on Antennas and Propagation, Vol. 59, No. 3, 2011, pp. 767-775. doi:10.1109/TAP.2010.2103025

[5] Y. Dong and T. Itoh, "Realization of a Composite Right/ Left-Handed Leaky-Wave Antenna with Circular Polarization," 2010 Asia-Pacific Microwave Conference Proceedings, Yokohama, 7-10 December 2010, pp. 865-868.

[6] Y. Dong and T. Itoh, "Metamaterial-Inspired Broadband
Mushroom Antenna," 2010 IEEE Antennas and Propagation Society International Symposium, Toronto, 11-17 July 2010, pp. 1-4. doi:10.1109/APS.2010.5560998

[7] T. Ueda, G. Haida and T. Itoh, "Zeroth-Order Resonators with Variable Reactance Loads at Both Ends," IEEE Transmission on Microwave Theory and Techniques, Vol. 59, No. 3, 2011, pp. 612-618. doi:10.1109/TMTT.2010.2103087

[8] A. Sanada, C. Caloz and T. Itoh, "Zeroth-Order Resonance in Composite Right/Left Handed Transmission Line Resonators," IEEE Transactions on Microwave Theory and Techniques, Vol. 59, No. 3, March 2003, pp. 1588-1592. 